\title{
Analysis of Fitness Value of Yoga and Its Marketization in the New Era
}

\author{
$\operatorname{Min} \mathrm{Li}$ \\ Jiangxi Science \& Technology Normal University, Nanchang, 330013, China
}

Keywords: Fitness value, Marketization, Management system

\begin{abstract}
This paper explores the introduction of the value of yoga and the existing problems of the Yoga market, finding that the effect of yoga is not understood by the market. The main problem is that the marketing and management system have hindered the development of yoga. We put forward some measures to accelerate the supervision of the system construction, improve the quality of the practitioners and the degree of social recognition to promote the better and faster development of the Yoga market.
\end{abstract}

\section{Introduction}

Since the thirty years of reform and opening, China's economy has entered a new stage of rapid development. As the second largest country of GDP in China, the richer and richer Chinese are increasingly not satisfied with the material needs, but gradually turn to concern about their physical condition, mental health and more noble pursuit. At this time, yoga was introduced from India to China, which basically satisfied people's pursuit of physical health and mental health in the new era, so it became more and more popular in China's fitness market. Breathing method, fixed position method and brain meditation yoga is the three most representative activities, it can effectively relax people tired one day, and let the spirit of tight pressure of urban life has been released, and with so much ease function of yoga fitness pursued. Through searching for a lot of data and literature, the author puts forward the necessity of Yoga market development by elaborating and deliberating repeatedly. By elaborating the value of yoga fitness, I hope to promote yoga in fitness business and promote its great development.

\section{Analysis of Fitness Value of Yoga}

In the ancient India Sanskrit, the word "Yoga", which means the original motivation of things and the organic combination of self, is recognized as the original source of yoga. Unlike previous sports and fitness programs, yoga is more concerned with physical and psychological performance. Yoga hopes to improve human will, to improve the physical condition, emotional state, mental state and physical condition of people.

Yoga is like the drawing and twisting action is very gentle, with individual bones stiff, spine is not straight, pelvis and shoulders are not inequality symptoms of fitness enthusiasts, can get a better improvement through yoga without losing the sense of beauty, but also to achieve the shape and lifting effect of temperament. In terms of body bonding, yoga exercises improve the emotional stability of the exercisers by focusing on static meditation, breathing and brain meditation.

Fixed position adjustment method, pranayama and meditation Yoga brain constitute the three-plate method, the fitness can be adjusted by breathing, posture change action and control the mental imagery to complete the yoga exercise, we can reach the inner gland secretion, visceral massage, relax muscles and nerves to. Unique posture and breathing method, combined with the spirit of Yoga emphasizes meditation can improve people fatigue mitigation capability and stress adjustment ability, achieve human and nature and thus strengthen the effect all blend into one harmonious whole, individual body shape and inner toughness and smooth meridian circulation. Studies have shown that many human diseases such as high blood pressure, hyperglycemia and 
hyperlipidemia in the three high levels. Amy shape, such as measurements in waist circumference, chest circumference, hip circumference can be effectively improved by yoga. Therefore, in the new era, people's rhythm of life is accelerating and living standards are improving. The indoor yoga exercise with simple and effective way is more and more liked and accepted by people.

\section{Analysis of Yoga Marketization}

\subsection{Imperfect Management System}

For our country, the traditional fitness program is deeply rooted. The Yoga project has been introduced from India for a short time, and the supporting facilities are not in place. Therefore, there is a lack of professional ability of management institutions. As the market management mechanism is not perfect, will not be able to do yoga industry scale and industry, yoga at home more exist in the private sector, and the absence of management problems and reverse lead Yoga qualification, lack of capacity problems also exist, resulting in industrial development as well as the Yoga course two-way blocked. Similarly, due to these problems, in the process of Yoga marketization progress, we pay attention to business returns, and ignore the fitness value and exercise methods of private institutions. To gain private benefits, we blindly exaggerate the effectiveness and seize the market, which further hinder the process of Yoga marketization. Therefore, the development of the yoga industry is also subject to the development of the industry standards under the times.

\subsection{Knowledge Vacancy of Relevant Employees}

Employees know spread of yoga teaching methods are uneven levels of yoga industry lack of scientific and systematic problems. Improving the physical quality and mental quality of yoga fitness students is the main goal of yoga fitness institutions and managers, which is conducive to the healthy operation and management of Yoga institutions. For a fitness organization that starts the Yu Ga course, it is necessary to recommend fitness trainees $\mathrm{Yu}$ Ga practice with appropriate fitness methods. But for most Yoga institutions, the introduction of yoga fitness project, not a deep understanding of the yoga classes, more just to introduce the project effect to attract members, students are not expected to be suitable for training yoga doubt. At present, yoga practitioners professional, more sports teachers, students or class athlete's physical yoga enthusiasts, teaching level since school, seriously affected the development of yoga fitness program.

\subsection{Low Accreditation of Social Groups}

At this stage, the popularity of yoga is low. The main exercise group is only limited to young women, and it only occupies a very low proportion in the female group. This reflects the low degree of social recognition in yoga. These are related to the whole Yoga atmosphere in the whole country. The more people who participate in yoga exercise, the higher the popularity is, so that we can improve the recognition of the whole yoga industry, which is beneficial for more people to contact this project. Similarly, in terms of internal analysis of fitness agencies, compared with dancing, aerobics and other women's shaping sports, the tuition fee is higher, which also leads to the two-way spiral influence of social recognition, and its recognition is difficult to effectively improve.

\section{Strategies of Yoga Marketization}

\subsection{Strengthen Yoga Marketization Management}

Under the current market development situation, the whole yoga industry presents a privatization oriented state of individual operation. Therefore, governments should play a leading role in the whole market management of yoga. First, local governments can promote the establishment of yoga fitness industry associations or academic organizations, and then introduce the concept of science and system development into yoga fitness. Learn from foreign advanced experience, improve the concept of development and improve the training system for yoga, and then promote the management and 
development of the yoga industry market. Secondly, the author believes that the establishment of the system of management and synchronization of yoga industry market, the purpose is to regulate the fitness yoga curriculum agencies additional access standards, and further perfecting the yoga industry operation and management mechanism, to regulate all the yoga curriculum management main body behavior. Finally, we need to establish an external macro guidance and supervision mechanism, such as establishing functional departments to strengthen Yoga qualification examination and yoga instructor's qualification certificate, and so on, and improve the whole yoga industry's overall situation. Actively through the system, professional, supervision and service to promote the industrialization of yoga to create a good market atmosphere.

\subsection{Enhance Teaching Requirements of Yoga Course}

The core of Yoga marketization is the quality training of practitioners in the yoga industry. The relevant departments, such as culture department, sports department and business department, strengthen the supervision and management of yoga practitioners in the process of Yoga management. The management department can ask yoga practitioners to choose the Yoga textbooks designated by the market, and carry out yoga teaching activities and curriculum development activities according to the relevant regulations. At the same time, we should also emphasize that practitioners should strengthen mutual contacts and exchanges, provide references for curriculum development and curriculum teaching, and make clear points for attention in teaching and teaching process, to provide better service guidance for yoga teaching activities. At the same time, the education department can also add yoga courses to all kinds of institutions including physical education institutes, and constantly strengthen the training and team building of teachers.

\subsection{Do a Good Job of Yoga Propaganda and Promotion}

The economic living conditions of the people of a country, including the degree of physical health and the degree of mental health, can also reflect the comprehensive strength of the country to a certain extent. Related agencies can vigorously promote yoga fitness under all kinds of offline media and online media, and adopt different measures to improve their cognition of yoga for different groups, to improve social recognition. At the same time, the relevant departments, universities and other institutions can be combined to carry out social promotion of yoga, yoga to carry out experiential marketing means, can also be yoga with other similar dance movement combined with combination of advance, increase its use and promote social acceptance.

\section{Conclusion}

The value of yoga is self-evident, and more and more people are coming into the ranks. The government and related agencies should solve several key marketing problems in the process of Yoga promotion, and integrate the market, institutions and practitioners into an organic whole, so as to promote the healthy development of the yoga industry.

\section{References}

[1] Chen Xiaoying. Study on the Value of Body- building and Marketization of Yoga [J]. Journal of Guangzhou Sport University, 2010,39(2): 112-115.

[2] Li Zhuojia, Lei Xuehui. Modern Value of Yoga [J]. Journal of Sports Adult Education, 2016, 32(3): 78-79.

[3] Li Zhenzhen. Value of Fitness Education of Yoga Course from the Perspective of Life Philosophy [J]. Journal of Huaibei Normal University (Natural Science Edition), 2015, 36(1): 70-72.

[4] Zhu Fang, Yue Minmin. A Preliminary Study of the Diversified Cultural Value of Contemporary Yoga [J]. Journal of Shandong Normal University (Natural Science), 2017, 32(3): 155-158. 